\title{
Giant Transversal Particle Diffusion in a Longitudinal Magnetic Ratchet
}

\author{
Pietro Tierno, ${ }^{1, *}$ Peter Reimann, ${ }^{2}$ Tom H. Johansen, ${ }^{3}$ and Francesc Sagués ${ }^{1}$ \\ ${ }^{1}$ Departament de Química Física, Universitat de Barcelona, Martí i Franquès 1, 08028 Barcelona, Spain \\ ${ }^{2}$ Fakultät für Physik, Universität Bielefeld, 33615 Bielefeld, Germany \\ ${ }^{3}$ Department of Physics, University of Oslo, P.O. Box 1048, Blindern, Norway
}

(Received 7 September 2010; published 1 December 2010)

\begin{abstract}
We study the transversal motion of paramagnetic particles on a uniaxial garnet film, exhibiting a longitudinal ratchet effect in the presence of an oscillating magnetic field. Without the field, the thermal diffusion coefficient obtained by video microscopy is $D_{0} \approx 3 \times 10^{-4} \mu \mathrm{m}^{2} / \mathrm{s}$. With the field, the transversal diffusion exhibits a giant enhancement by almost four decades and a pronounced maximum as a function of the driving frequency. We explain the experimental findings with a theoretical interpretation in terms of random disorder effects within the magnetic film.
\end{abstract}

PACS numbers: 05.40.- a, 02.50.Ey, 05.45.- a, 05.60.-k

Transport and diffusion in periodic or random potentials play a key role in many different contexts of physics, chemistry, and biology [1,2]. Most prominently, directed transport by ratchet effects has been scrutinized in a huge number of theoretical works [1] due to its fascinating perspectives with respect to basic statistical physics, intracellular transport, and technological applications. Also, the experimental literature has substantially grown in recent years, demonstrating ratchet effects for colloidal particles [3], Abrikosov vortices [4] and Josephson vortices [5] in superconductors, electrons in semiconductor heterostructures [6], Josephson phases across SQUIDs [7], cold atoms [8], granular gases [9], single cells [10], and various magnetic systems [11]. A related magnetic ratchet effect is also at the basis of our present work: The parallel magnetic stripes (domains) of a ferrite garnet film (FGF) are externally modulated by an oscillating magnetic field, resulting in a deterministic ratchet effect of paramagnetic particles in the form of directed transport perpendicularly to the stripes. The main result is the observation that this ratchet effect triggers a giant enhancement of the transversal (parallel to the stripes) diffusion of the particles, exceeding their field-free diffusion by almost four decades. Moreover, by varying the oscillation frequency, the transversal diffusion coefficient exhibits a pronounced peak. This anomalous behavior is explained in terms of an analytical model. In contrast to enhanced diffusion in (effectively) onedimensional models [12] and experiments [13], this effect is only possible in two dimensions and is governed by a completely different physical mechanism. Also Galtonboard-type systems [14] are very different from ours.

In our experiments we use polystyrene paramagnetic particles (radius $a=1.4 \mu \mathrm{m}$, volume susceptibility $\chi=$ 0.4 , Dynabeads M-270), diluted in deionized water (negligible particle-particle interaction), and moving on top of an epitaxial grown FGF with uniaxial anisotropy [15]. As sketched in Fig. 1(a), the FGF exhibits periodic stripes of magnetic domains with alternating up and down magnetization direction (saturation magnetization $M_{s}=$ $1.7 \times 10^{4} \mathrm{~A} / \mathrm{m}$, spatial periodicity $\left.\lambda=6.9 \mu \mathrm{m}\right)$. The domains are separated by Bloch walls (BWs), where the magnetic stray field $\boldsymbol{H}_{s}$ of the FGF is maximal. Application of a spatially uniform oscillating field with inclination $\vartheta$ in the $x-z$ plane, $\boldsymbol{H} \equiv H \sin (\omega t) \times$ $(\sin \vartheta, 0, \cos \vartheta)$, has two main effects: First, the $z$ component of $\boldsymbol{H}$ displaces the BWs by increasing (decreasing) the width of the domains with parallel (opposite) magnetization direction. The $x$ component of $\boldsymbol{H}$ breaks the symmetry of the potential as the BW array becomes an alternating sequence of strong and weak pinning sites according to where the local stray field is parallel or antiparallel to the in-plane applied field, respectively; see Fig. 1(a). Second, a paramagnetic particle acquires a magnetic moment $\boldsymbol{m}=$ $\left(4 a^{3} \pi / 3\right) \chi\left(\boldsymbol{H}+\boldsymbol{H}_{s}\right)$, entailing a magnetic force $\boldsymbol{F}=$ $\mu \nabla\left(\left(\boldsymbol{H}+\boldsymbol{H}_{s}\right) \cdot \boldsymbol{m}\right)$, where $\mu$ is the magnetic susceptibility of the medium. As detailed in [16], the particle motion on top on the FGF is basically confined to the $x-y$ plane and the net force along the $x$ axis derives from a potential $V(x, t)$ as indicated in Fig. 1(b), while the forces in the $y$ direction are negligible in a first approximation. The spatially asymmetric ratchet potential $V(x, t)$ gives rise to a ratchet effect [1], "dragging" along the particles in a deterministic way (thermal noise is negligible) by one spatial period $\lambda$ in the $x$ direction during one driving period $2 \pi / \omega$. We experimentally measure a maximum driving frequency of $\omega_{\max } \approx$ $200 \mathrm{~s}^{-1}$, beyond which the overdamped particles cannot follow the fast modulations.

Particles are tracked with a light microscope (E400, Nikon) equipped with a CCD camera (Basler) working at 60 frames per second. After recording $N$ independent trajectories $\left\{x_{n}(t), y_{n}(t)\right\}_{n=1}^{N}$, we measure the transversal diffusion coefficient in the long-time limit, $D_{y}=$ $\lim _{t \rightarrow \infty}\left\langle[y(t)-\langle y(t)\rangle]^{2}\right\rangle / 2 t$, where $\langle\cdots\rangle$ denotes an average over independent experiments.

Without external field $\boldsymbol{H}$, the particles are pinned by the BWs, resulting in transversal thermal diffusion, while 

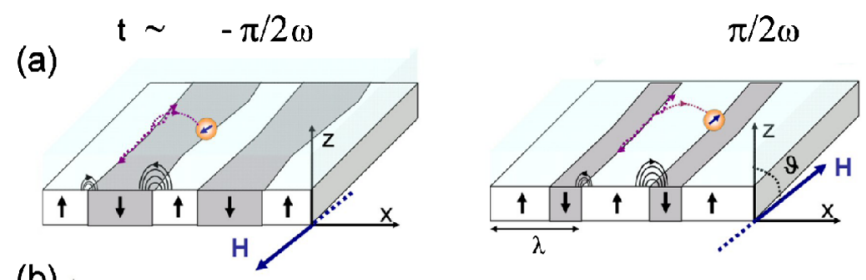

(b)

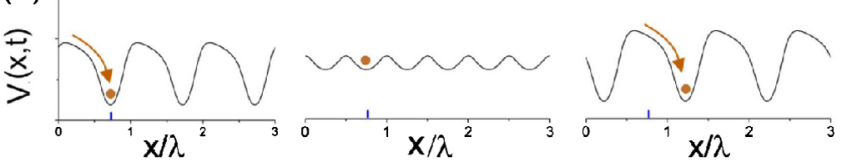

(c)

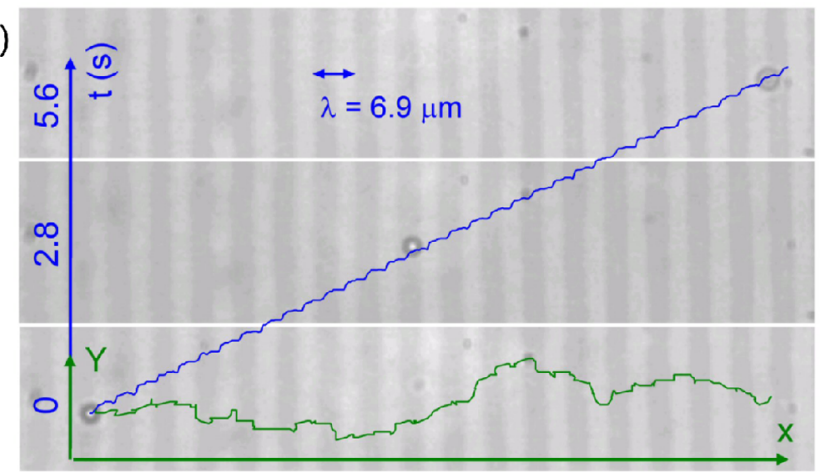

FIG. 1 (color online). (a) Schematic illustrations of a traveling paramagnetic particle on top of the FGF, the stray field of the BWs, and the oscillating external field $\boldsymbol{H}$ for two different times $t=-\pi / 2 \omega$ and $t=\pi / 2 \omega$. (b) Corresponding potentials $V(x, t)$ (arb. units) entailing particle motion in $x$ direction and calculated from Eq. (1) of Ref. [16]. The middle graph corresponds to $H=0$. (c) Gray: Three optical microscope snapshots of the FGF and the traveling particle at $t=0,2.8$, and $5.6 \mathrm{~s}\left(\omega=18.8 \mathrm{~s}^{-1}\right.$, $H=1500 \mathrm{~A} / \mathrm{m}, \vartheta=\pi / 7$ ). Black (blue) line: Corresponding particle trajectory $x(t)$. Gray (green) line: Corresponding path in the $x-y$ plane. See also Video2 in [17].

longitudinal excursions from the BWs are excluded (Video1 in [17]). The quantitative details are provided with Fig. 2, yielding an unperturbed transversal diffusion coefficient of $D_{0}=3.2 \times 10^{-4} \mathrm{\mu m}^{2} / \mathrm{s}$, which is almost three decades smaller than the diffusion coefficient $D_{\mathrm{sf}}=$ $0.09 \mu \mathrm{m}^{2} / \mathrm{s}$ measured on a stripe-free FGF [18].

Upon application of the oscillating field $\boldsymbol{H}$, the particles exhibit the above-mentioned ratchet effect in the $x$ direction. The concomitant motion in the $y$ direction shows the typical qualitative features of an unbiased random walk [Fig. 1(c), Video2 in [17]). Figure 2 confirms quantitatively that we are indeed dealing with a standard (normal) diffusion process [19], with a mean square displacement characterized by a power law behavior with exponent $\alpha=1$ for large $t$. Striking enough, this diffusion coefficient $D_{y}$ exhibits a pronounced peak as a function of the driving frequency $\omega$, exceeding the unperturbed diffusion $D_{0}$ by up to almost orders of magnitude; see Fig. 3. The rest of the Letter is devoted to the explanation of these findings.

According to Fig. 1, a particle performs a jump from one BW to the next during every half-period $\tau=\pi / \omega$ of the

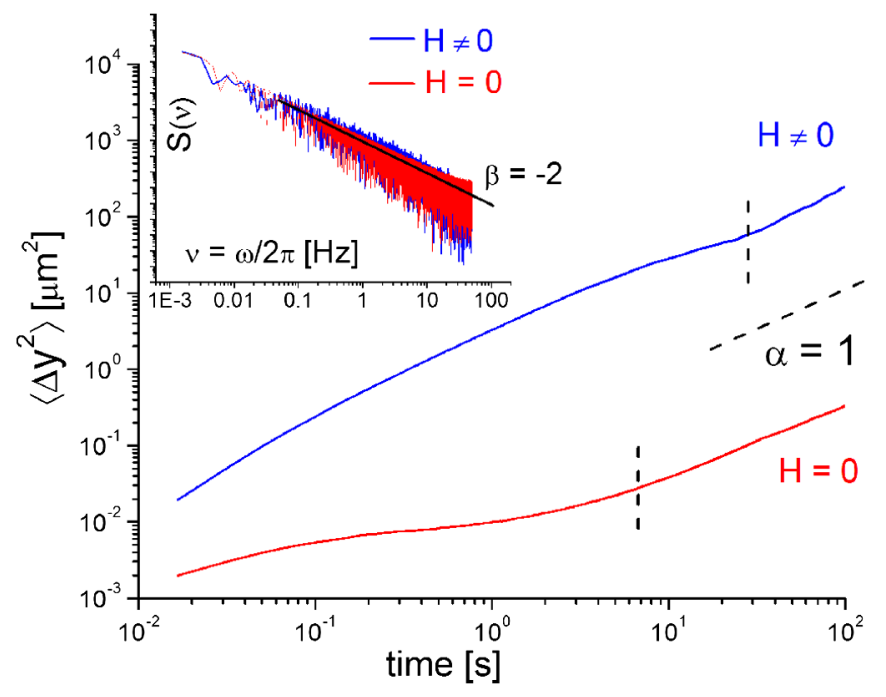

FIG. 2 (color online). Transversal mean square displacement $\left\langle\Delta y^{2}\right\rangle$ versus time (log-log-plot). Black (blue) line: Experimental results for the same system as in Fig. 1. Gray (red) line: Same but without external field. The approach of a straight line with slope $\alpha=1$ for large times indicates convergence in $D_{y}$; the curves starting from the dashed lines were used to calculate $D_{y}$. Inset: The power spectra $S(\nu):=|Y(\nu)|^{2}$, where $Y(\nu)$ is the Fourier transform of one representative trajectory $y(t)$. The convergence towards a straight line with slope $\beta=-2$ for small $\nu$ indicates normal (as opposed to anomalous) diffusion [19].

driving. Furthermore, each jump is initiated by the progressive disappearance of the potential well pinning the particle and is completed by the subsequent relaxation towards a new potential minimum. For driving frequencies $\omega$ well below the threshold $\omega_{\max }$, the duration $\tau_{j}$ of a jump is much smaller than the half-period $\tau$ and turns out to be approximately independent of $\omega$. In turn, for $\omega$ exceeding $\omega_{\max }$, a jump would take more than the total available halfperiod $\tau$ and hence the entire ratchet mechanism from Fig. 1 breaks down. These considerations suggest the rough estimate $\tau_{j} \approx \pi / \omega_{\max }=0.02 \mathrm{~s}$, in very good agreement with the experimental data from Fig. 4. Altogether, within every half-period $\tau$, the particle thus moves along one and the same BW during an exploration time of length $\tau_{e}=\tau-\tau_{j}$, and then jumps to the next BW.

Within the diffraction limit ( $\approx 200 \mathrm{~nm}$ ) of our optics, the BWs appear as practically straight border lines in Fig. 1(c). However, it is known that on smaller scales they do exhibit notable random undulations, caused by pinning sites and other inhomogeneities of the FGF. As shown in Ref. [20], the amplitude and bend period of the undulations are not reproducible upon reapplication of the field. As a consequence, the random undulations of different BWs are independent of each other. Because of similar reasons, the magnetic stray field, experienced by a particle when moving along a BW, also exhibits random variations.

The simplest quantitative modeling consists in assuming an overdamped relaxation dynamics $\eta \dot{y}(t)=-U^{\prime}(y(t))$, 

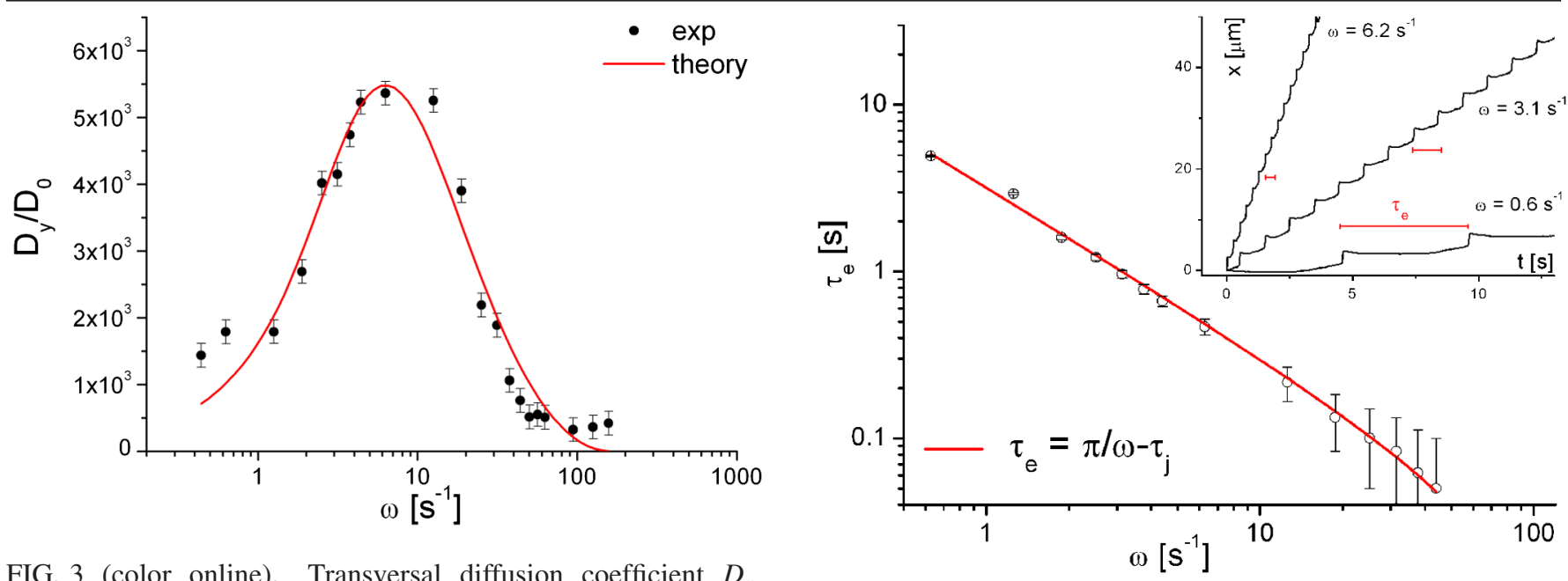

FIG. 3 (color online). Transversal diffusion coefficient $D_{y}$ versus driving frequency $\omega$ in units of $D_{0}=3.2 \times$ $10^{-4} \mu \mathrm{m}^{2} / \mathrm{s}$ (undriven diffusion). Dots: Experimental findings for the same system as in Fig. 1. Line: Theoretical approximation (1) with fit parameters $\sigma=1.8 \pm 0.2 \mu \mathrm{m}$ and $k=2.9 \pm$ $0.1 \mathrm{~s}^{-1}$

where $U(y)$ is a random magnetic potential, negligibly weak with regard to jumps between the BWs ( $x$ direction), but not with regard to the dynamics in $y$ direction (along the BWs). Thermal noise is neglected in view of our experimental finding that the field-free diffusion $D_{0}$ along a BW is much smaller than thermal diffusion $D_{\text {sf }}$ on a stripe-free FGF. The damping coefficient $\eta$ can be estimated via Einstein's formula $D_{\text {sf }}=k_{B} T / \eta$, with $k_{B}$ Boltzmann's constant and $T \approx 300 \mathrm{~K}$, yielding $\eta \approx$ $0.05 \mathrm{mg} / \mathrm{s}$. Jumps between BWs are modeled by resetting or resampling the random potential $U(y)$, while keeping $y(t)$ fixed during the corresponding jump time $\tau_{j}$. To be specific, we focus on the simplest possible example, namely, a potential $U(y)$ composed of parabolic pieces, each piece (index $i$ ) exhibiting the same curvature $\kappa>0$, but with randomly sampled extensions to the right and to the left of its minimum $y_{i}$. Thus a seed $y(0)$ within the $i$ th parabolic piece evolves according to $\dot{y}(t)=-k\left(y(t)-y_{i}\right)$ with $k:=\kappa / \eta$. For the displacement after half a period $\tau$, one thus obtains $y(\tau)-y(0)=\xi\left(1-e^{-k \tau_{e}}\right)$ with $\xi:=$ $y_{i}-y(0)$. Similarly, one obtains for the displacement after $P$ half-periods $y(P \tau)-y(0)=\left(1-e^{-k \tau_{e}}\right) \sum_{p=1}^{P} \xi_{p}$, where the resampling of the random potential $U(y)$ after each jump implies that the $\xi_{p}$ are independent, identically distributed random numbers of zero average and a finite variance $\sigma^{2}$. This leads to

$$
D_{y}=\frac{\sigma^{2}}{2 \tau}\left[1-e^{-k\left(\tau-\tau_{j}\right)}\right]^{2},
$$

where $\sigma$ and $k$ are fit parameters. The former can be readily identified with the characteristic length scale of the BW undulations, while $k^{-1}$ gives the relaxation time scale. Figure 3 demonstrates very good agreement of (1) with

FIG. 4 (color online). Exploration time $\tau_{e}$ versus driving frequency $\omega$. Circles: Experimental results. Line: Theoretical approximation $\tau_{e}=\pi / \omega-\tau_{j}$ with $\tau_{j}=0.02 \mathrm{~s}$. Inset: Experimental particle trajectories $x(t)$ for different driving frequencies $\omega . \tau_{e}$ (red bars) indicates the exploration time between two jumps in $x$ direction, during which the particle moves along one given BW.

the experimental observations, with $\sigma$ being reasonably of the order of the domain width $(\lambda / 2)$ and $k^{-1}$ matching the time scale of the applied forcing when a significant enhancement of $D_{y}$ occurs. Admitting more than a single curvature of the parabolic pieces yields an analytical result containing (1) as a special case, and thus fitting the experiment even better. Further analytical solutions are possible for piecewise sinusoidal instead of piecewise parabolic random potentials $U(y)$, fitting the experiment practically equally well. The details of $U(y)$ thus seem to matter very little.

For small $\omega$, the asymptotics $D_{y} \sim \omega$ of (1) reflects the fact that the particles have ample time to relax into the next potential minimum along every $\mathrm{BW}$, but then have to await the next jump. Conversely, for $\omega \rightarrow \omega_{\max }$, the particles have almost no time to explore the BWs and thus $D_{y} \sim$ $\left(\omega_{\max }-\omega\right)^{2}$ according to (1). It follows that there must be some intermediate $\omega$ at which $D_{y}$ exhibits a maximum. The vanishing of (1) at $\omega=\omega_{\max }$ is nonrealistic, and is due to neglecting any motion in the $y$ direction during the jump times $\tau_{j}$. Within our model, the field-free diffusion $D_{0}$ corresponds to a single, fixed random potential $U(y)$, and the stripe-free diffusion $D_{\text {sf }}$ to a constant $U(y)$.

Experimentally, variations of the driving amplitude $H$ were restricted to the regime $0.06 M_{s}<H<0.15 M_{s}$. Within this regime, $D_{y}$ did not notably depend on $H$, in agreement with the theory. For smaller $H$, the particles were not able to jump, while larger $H$ led to irreversible deformations of the stripes and inhibition of particle hopping between BWs. We also explored the effect of high bending stripe deformation (i.e., "zigzag" stripe patterns), which leads to a counterintuitive decrease of the 
transversal diffusion coefficient $D_{y}$ since the particles funnel into narrow trajectories (see Fig. 2 in [17]). The natural explanation within our model follows by superimposing to the random potentials $U(y)$ one and the same periodic potential for all $\mathrm{BWs}$, taking into account that they all exhibit the same zigzag pattern.

To conclude, we observed giant transversal diffusion of paramagnetic colloidal particles on a garnet film, triggered by a longitudinal external driving and a concomitant longitudinal ratchet effect. We interpret this as the signature of a field induced undulation instability in the magnetic domain structures, invoking the presence of a time-refreshed disordered potential landscape in the magnetic pattern. The understanding and control of diffusion along the lines of our present work is a key issue in many technological and biophysical contexts [12-14].

We acknowledge discussions with J.M. Sancho and T.M. Fischer. P.T. was supported by the program "Juan de la Cierva" (JCI-200904192). P. T. and F. S. acknowledge financial support by MEC (FIS2006-03525) and DURSI (2009SGR1055), P.R. by Deutsche Forschungsgemeinschaft (SFB 613), and T.H.J. by The Research Council of Norway.

\section{*ptierno@ub.edu}

[1] F. Jülicher, A. Ajdari, and J. Prost, Rev. Mod. Phys. 69, 1269 (1997); P. Hänggi and F. Marchesoni, ibid. 81, 387 (2009); P. Reimann, Phys. Rep. 361, 57 (2002).

[2] J.-P. Bouchaud and A. Georges, Phys. Rep. 195, 127 (1990); H. Risken, The Fokker-Planck Equation (Springer, Berlin, 1984), Chap. 11; P. Reimann et al., Phys. Rev. E 65, 031104 (2002), and references therein.

[3] J. Rousselet et al., Nature (London) 370, 446 (1994); L. P. Faucheux et al., Phys. Rev. Lett. 74, 1504 (1995); C. Marquet et al., ibid. 88, 168301 (2002); L. R. Huang et al., ibid. 89, 178301 (2002); S.-H. Lee et al., ibid. 94, 110601 (2005); K. Loutherback et al., ibid. 102, 045301 (2009).

[4] J.E. Villegas et al., Science 302, 1188 (2003); J. Van de Vondel et al., Phys. Rev. Lett. 94, 057003 (2005); Y. Togawa et al., ibid. 95, 087002 (2005); C. C. de Souza Silva et al., ibid. 98, 117005 (2007); W. Gillijns et al., ibid. 99, 247002 (2007); B. B. Jin et al., Phys. Rev. B 81, 174505 (2010).

[5] J. B. Majer et al., Phys. Rev. Lett. 90, 056802 (2003); A. V. Ustinov et al., ibid. 93, 087001 (2004); D. E. Shalóm and H. Pastoriza, ibid. 94, 177001 (2005); M. Beck et al., ibid. 95, 090603 (2005).

[6] H. Linke et al., Science 286, 2314 (1999); E. M. Höhberger et al., Appl. Phys. Lett. 78, 2905 (2001).
[7] A. Sterck, R. Kleiner, and D. Koelle, Phys. Rev. Lett. 95, 177006 (2005); A. Sterck, D. Koelle, and R. Kleiner, ibid. 103, 047001 (2009).

[8] C. Mennerat-Robilliard et al., Phys. Rev. Lett. 82, 851 (1999); P. H. Jones, M. Goonasekera, and F. Renzoni, ibid. 93, 073904 (2004); P. Sjölund et al., ibid. 96, 190602 (2006).

[9] D. van der Meer et al., Phys. Rev. Lett. 92, 184301 (2004); P. Eshuis et al., ibid. 104, 248001 (2010).

[10] G. Mahmud et al., Nature Phys. 5, 606 (2009); R. Di Leonardo et al., Proc. Natl. Acad. Sci. U.S.A. 107, 9541 (2010); A. Sokolov et al., Proc. Natl. Acad. Sci. U.S.A. 107, 969 (2009).

[11] T. Deng et al., Appl. Phys. Lett. 78, 1775 (2001); A. Engel et al., Phys. Rev. Lett. 91, 060602 (2003); P. Tierno, T. H. Johansen, and T. M. Fischer, Phys. Rev. Lett. 99, 038303 (2007); P. Dhar et al., J. Phys. Chem. B 111, 13097 (2007); P. Tierno et al., ibid. 112, 3833 (2008); A. Pérez-Junquera et al., Phys. Rev. Lett. 100, 037203 (2008); A. Auge et al., Appl. Phys. Lett. 94, 183507 (2009).

[12] H. Gang, A. Daffertshofer, and H. Haken, Phys. Rev. Lett. 76, 4874 (1996); M. Schreier et al., Europhys. Lett. 44, 416 (1998); G. Costantini and F. Marchesoni, ibid. 48, 491 (1999); P. Reimann et al., Phys. Rev. Lett. 87, 010602 (2001); D. Reguera et al., Europhys. Lett. 57, 644 (2002); A. A. Dubkov and B. Spagnolo, Phys. Rev. E 72, 041104 (2005); B. Lindner and E. M. Nicola, Phys. Rev. Lett. 101, 190603 (2008); P. Reimann and R. Eichhorn, ibid. 101, 180601 (2008); P. Romanczuk, F. Müller, and L. Schimansky-Geier, Phys. Rev. E 81, 061120 (2010).

[13] S. H. Lee and D. G. Grier, Phys. Rev. Lett. 96, 190601 (2006); V. Blickle et al., Phys. Rev. E 75, 060101(R) (2007); G. Coupier et al., Europhys. Lett. 77, 60001 (2007); S. Bleil, P. Reimann, and C. Bechinger, Phys. Rev. E 75, 031117 (2007); M. Evstigneev et al., ibid. 77, 041107 (2008).

[14] C. Keller, F. Marquardt, and C. Bruder, Phys. Rev. E 65, 041927 (2002).

[15] P. Tierno et al., Phys. Chem. Chem. Phys. 11, 9615 (2009).

[16] P. Tierno et al., Phys. Rev. E 75, 041404 (2007).

[17] See supplementary material at http://link.aps.org/ supplemental/10.1103/PhysRevLett.105.230602 for supporting experimental images and videos.

[18] The same FGF under an external field $H>M_{s}$ such that the surface has no magnetic stripes.

[19] I. Golding and E. C. Cox, Phys. Rev. Lett. 96, 098102 (2006); R. Metzler and J. Klafter, Phys. Rep. 339, 1 (2000).

[20] P. Molho et al., J. Magn. Magn. Mater. 54-57, 857 (1986); D. Sornette, J. Phys. (Paris) 48, 151 (1987); V. A. Zablotskii, K. V. Lamonova, and Y. A. Mamalui, Physica (Amsterdam) 205B, 371 (1995). 